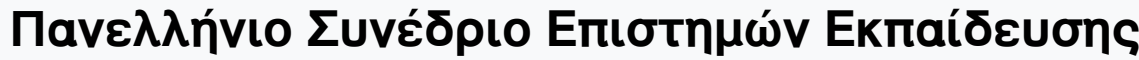

Tóp. 2016, Ap. 1

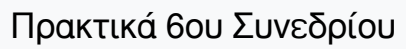

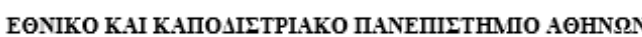

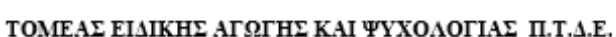

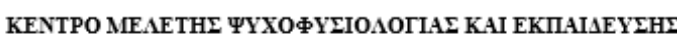

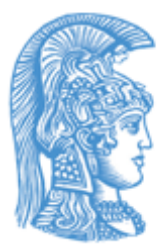

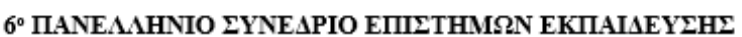

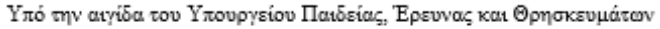

24-26 Iouviov 2016

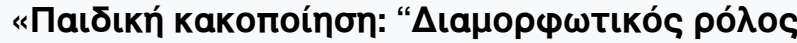

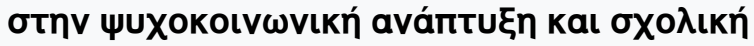

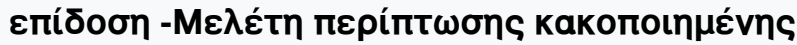
$\varepsilon \dot{\varphi \eta \beta \eta \varphi " ~}$

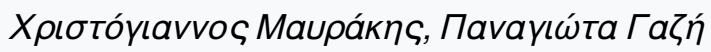

doi: $10.12681 /$ edusc.981

\section{ПIPAKTIKA 乏YNEAPIOY}

ISSN: 2529-1157

EIIMEAEIA:

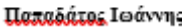

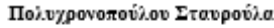

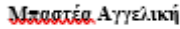

IOYNIOE 2016

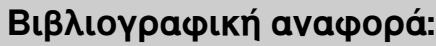

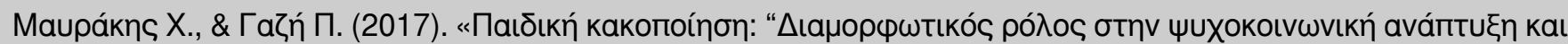

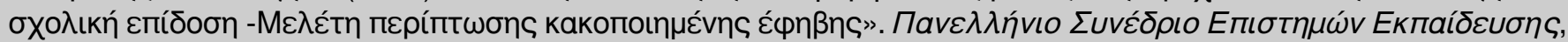
2016(1), 661-673. https://doi.org/10.12681/edusc.981 


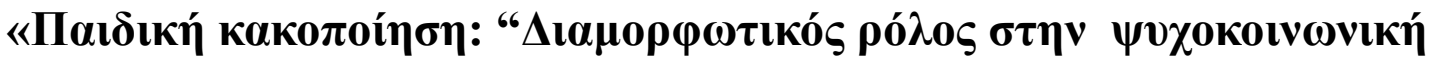

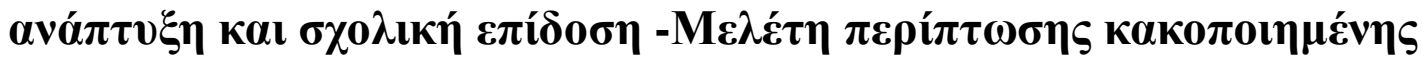

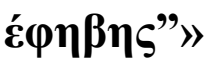

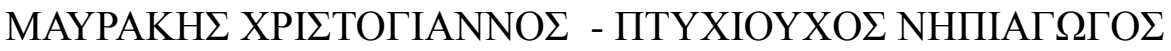

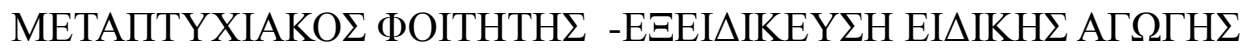

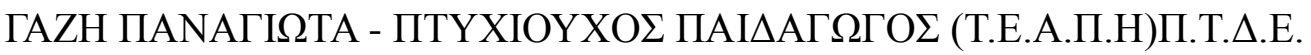

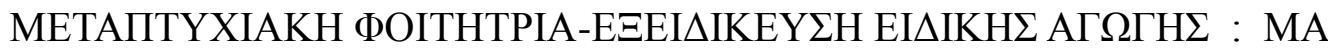 \\ Special Education Needs MBA ininternational Business
}

\section{Пєрí $\eta \psi \eta$}

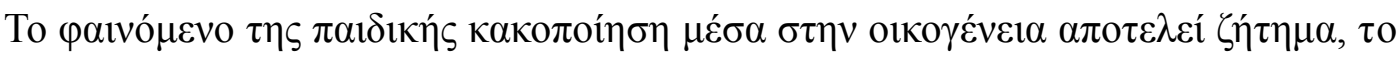

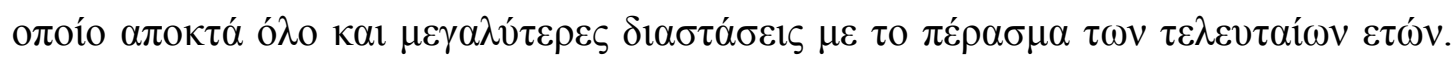

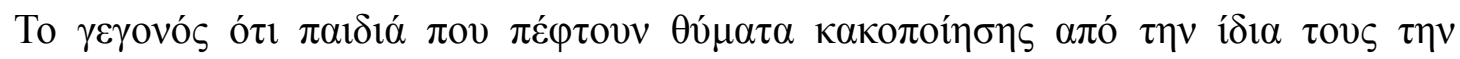

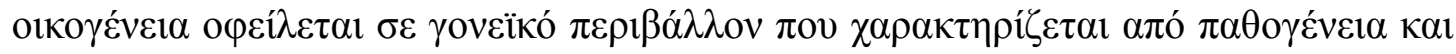

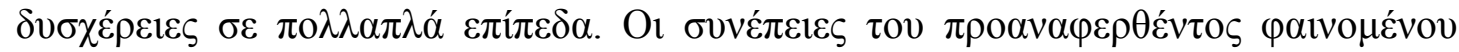

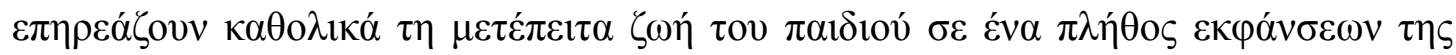

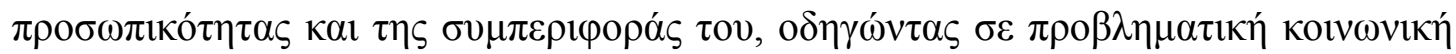

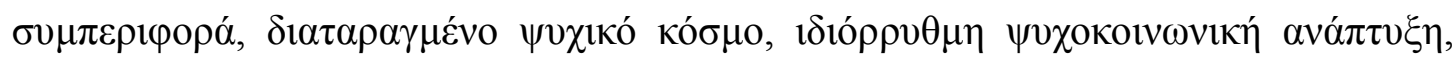

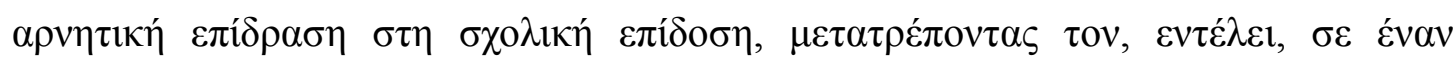

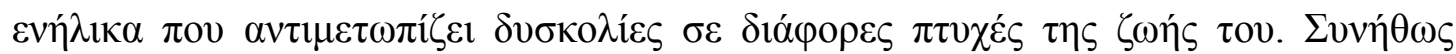

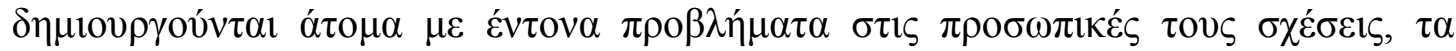

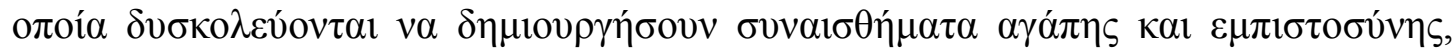

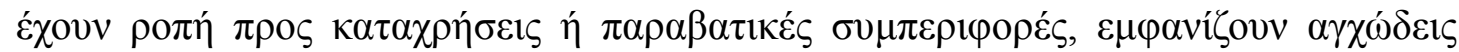

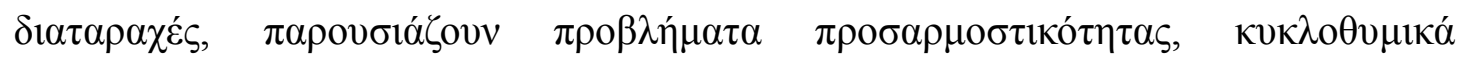

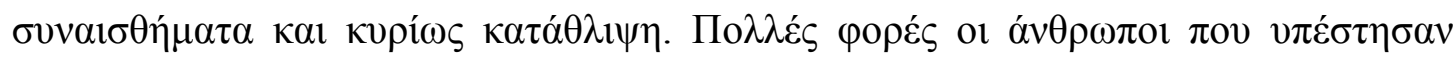

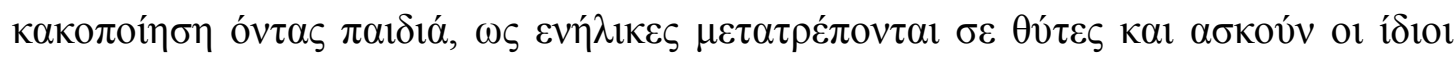

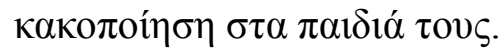

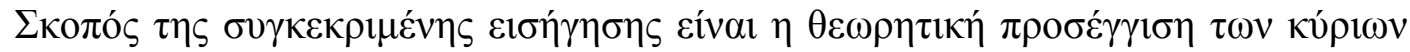

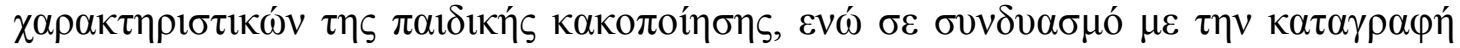

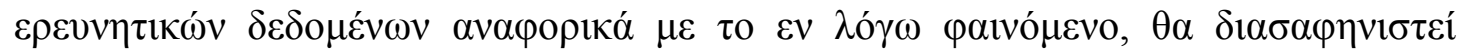

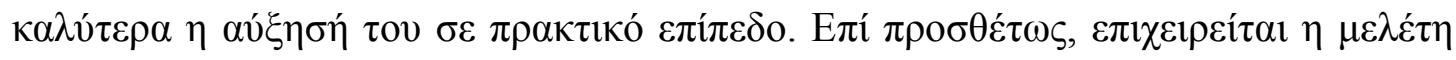




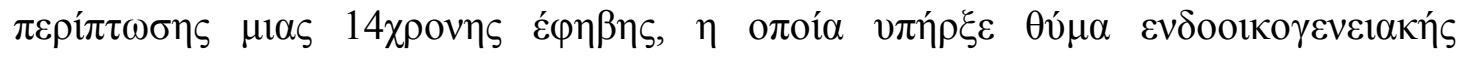

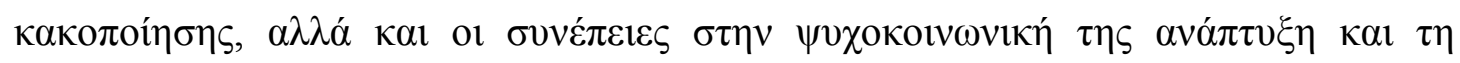

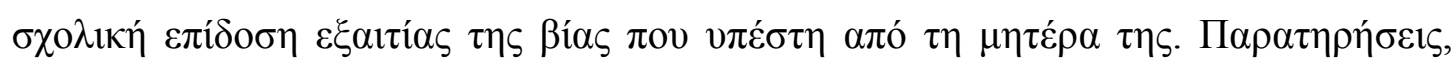

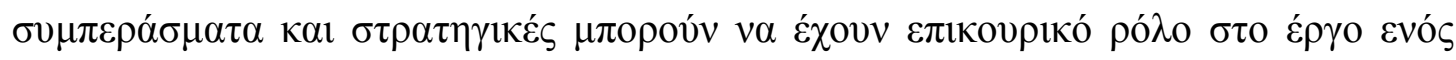

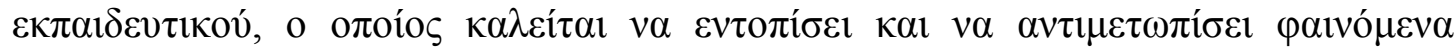

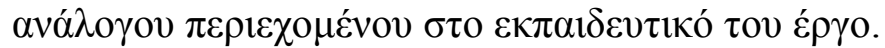

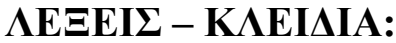

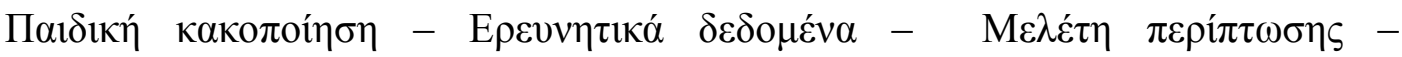

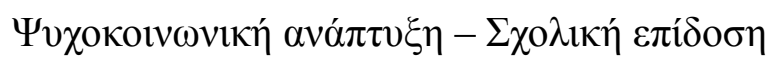

\begin{abstract}
The phenomenon of child abuse in the family environment consists an issue whose more and more aspects are enlightened over the last years. The fact that children become victims of abuse by their own family is due to parenthood characterized by pathogenesis and distress in multiple levels. The consequences of the already mentioned phenomenon affect the whole later life of a child as far as his personality and behavior are concerned, leading to problematic social treatment, disturbed mental balance, particular psychological development, negative impact to school performance, resulting finally in an adult who faces difficulties to all the aspects of his life. Most of the time, these adults have intense problems in their relationships with others, they have difficulties creating feelings of love and trust, they are prone to abuse of alcohol, drugs etc. or to delinquency, they have anxiety disorder, they cannot be adapted easily to an environment, they experience moody feelings and mainly depression. In a lot of cases, adults who have been abused during their childhood are turned into abusers to their own children.
\end{abstract}

The aim of this hypothesis is the theoretical approach of the main features of child abuse, as well as the practical clarification of its increase via the recording of research clues relevant to this phenomenon. Moreover, it is cited the case study of a fourteenyear-old girl who has been a victim of domestic abuse, and more specifically by the side of her mother. The consequences of the abuse to her psychosocial development and her school progress are also mentioned. Notices, conclusions and strategies are 
hoped to help all kind of instructors who are required to face this kind of phenomenon.

\section{KEY WORDS:}

Child abuse - Research Data - Case study - Psychosocial development- School performance

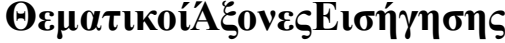

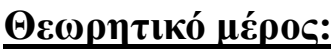

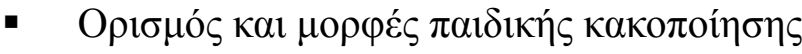

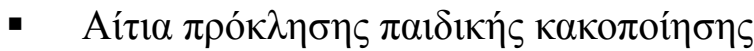

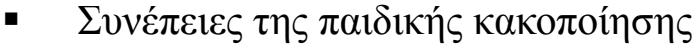

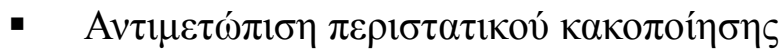

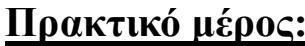

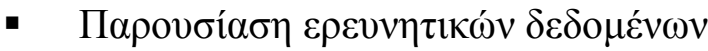

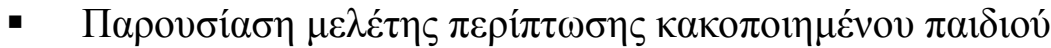

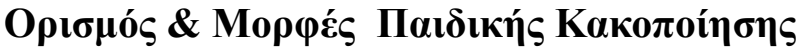

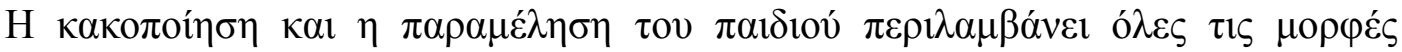

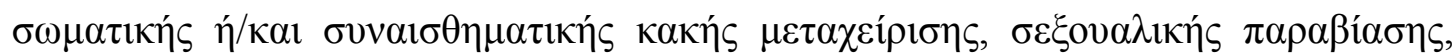

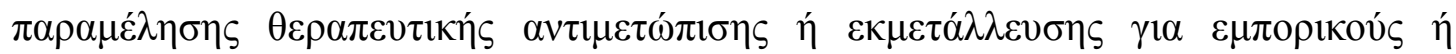

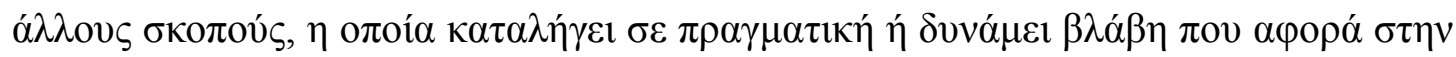

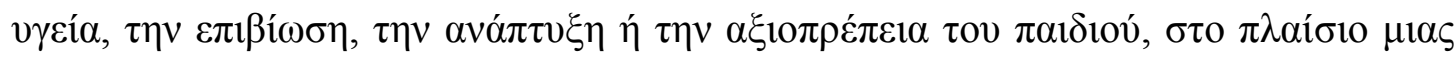

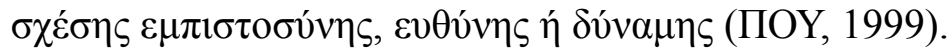

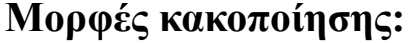

- $\sigma \omega \mu \alpha \tau 1 \kappa \eta ́$

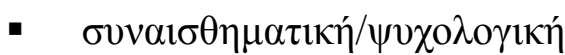

- $\sigma \varepsilon \xi o v \alpha \lambda \iota \kappa \eta ́$

- $\pi \alpha \rho \alpha \mu \varepsilon ́ \lambda \eta \sigma \eta$

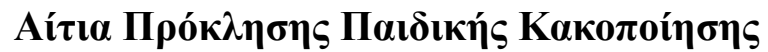




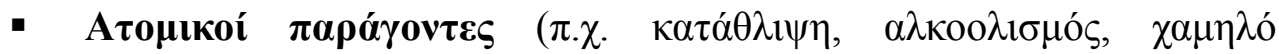


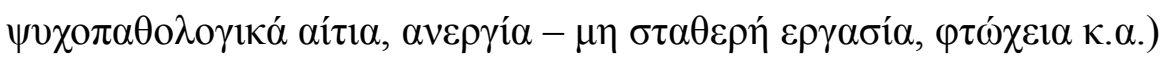

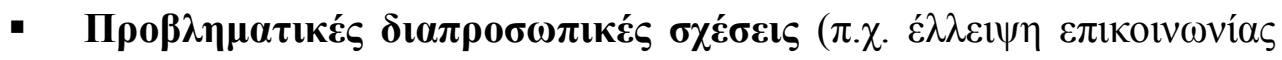

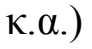

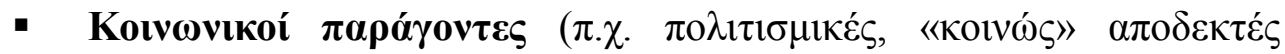

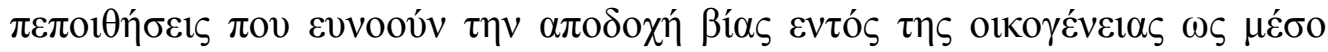
$\varepsilon \pi ı \beta о \lambda \eta ́(\varsigma \pi \varepsilon \imath \theta \alpha \rho \chi i ́ \alpha \varsigma)$

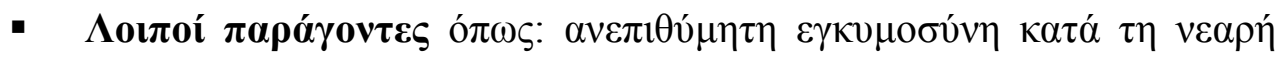

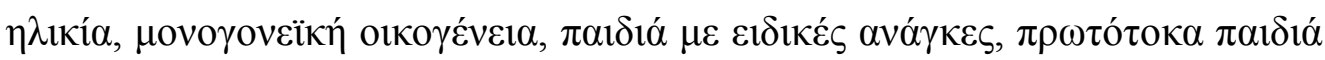
$\kappa . \alpha$.

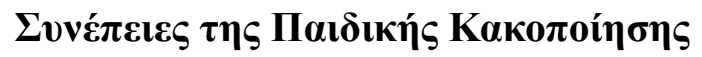

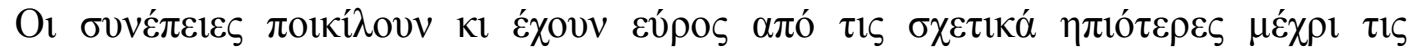

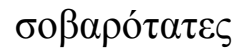
$\kappa \alpha 1$

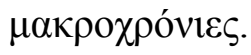

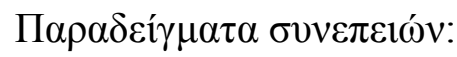

$\mu \alpha \theta \eta \sigma \iota \alpha \kappa \varepsilon ́ \varsigma \quad \delta v \sigma \kappa o \lambda i \varepsilon \varsigma, \quad \pi \rho \circ \beta \lambda \dot{\eta} \mu \alpha \tau \alpha \quad \sigma v \mu \pi \varepsilon \rho \imath \varphi о \rho \alpha \dot{\varsigma}, \quad \kappa \alpha \tau \dot{\alpha} \theta \lambda \imath \imath \eta, \quad \chi \alpha \mu \eta \lambda \dot{\eta}$

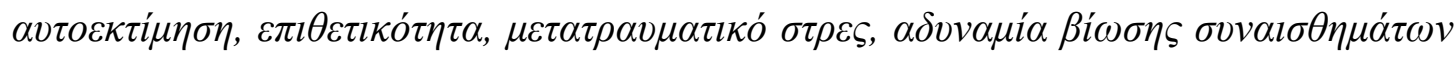

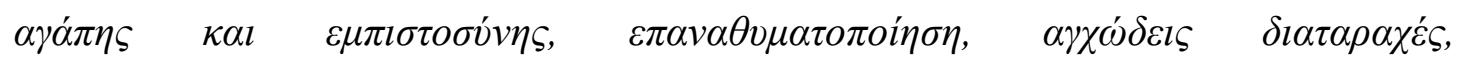


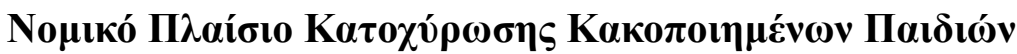

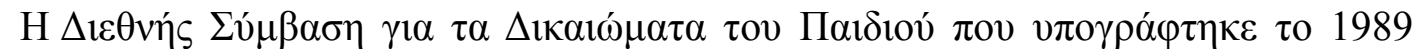

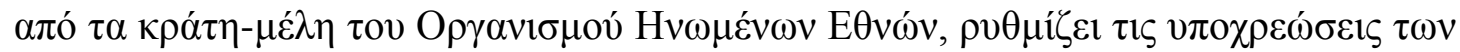

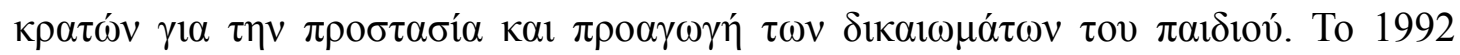

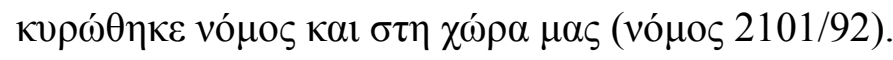

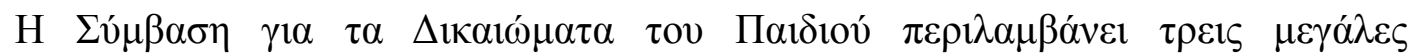

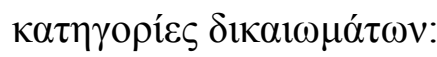

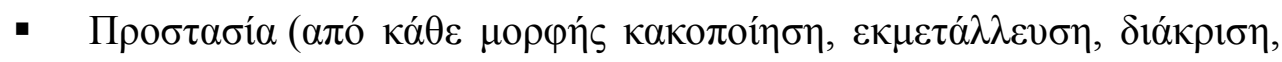
$\rho \alpha \tau \sigma \iota \mu \mu ́, \kappa . \lambda \pi$.) 


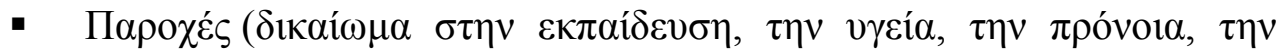
$\psi v \chi \alpha \gamma \omega \gamma i \alpha, \kappa . \lambda \pi$.)

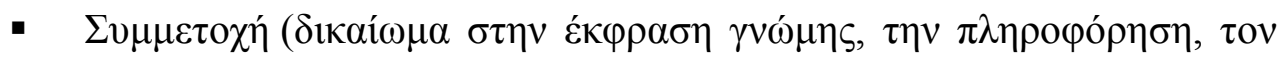

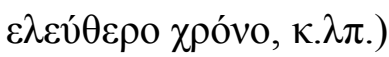

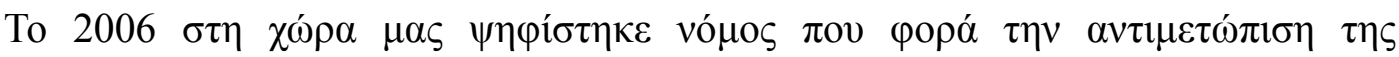

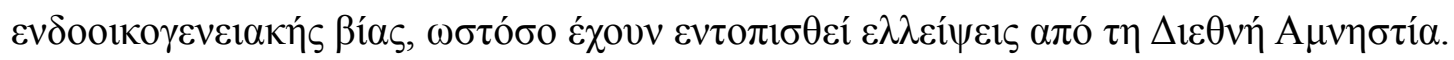

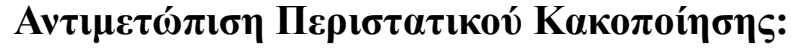

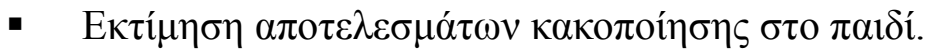

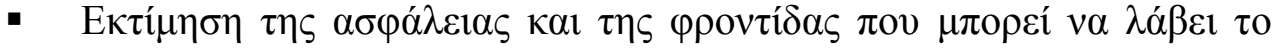

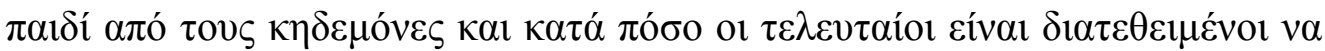

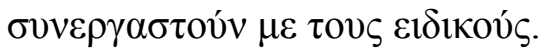

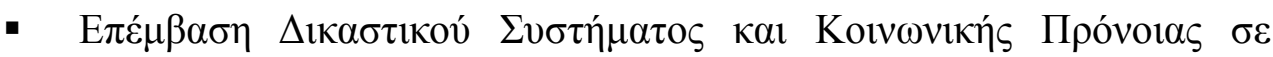

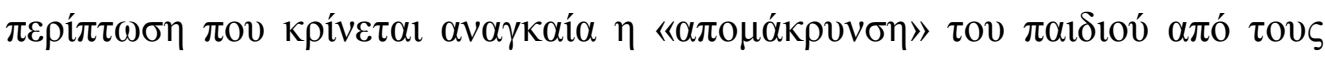
$\kappa \eta \delta \varepsilon \mu o ́ v \varepsilon \varsigma \tau o v$.

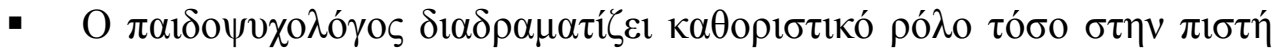

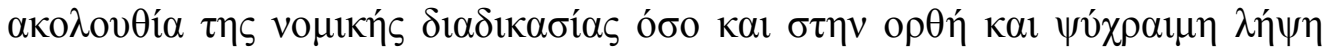

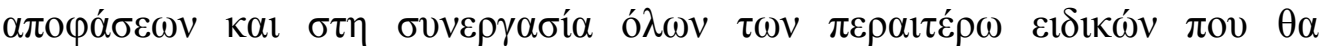
$\varepsilon \mu \pi \lambda \alpha \kappa o v ́ v \sigma \tau \eta \delta 1 \alpha \delta 1 \kappa \alpha \sigma i ́ \alpha$.

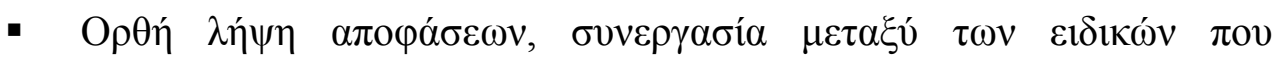

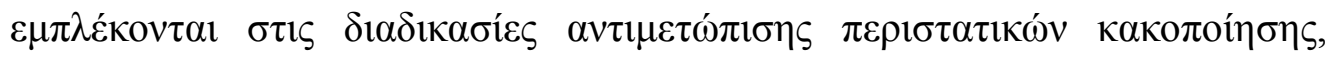

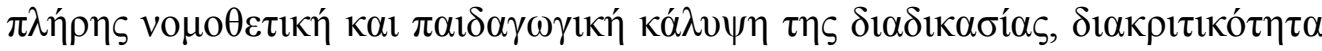

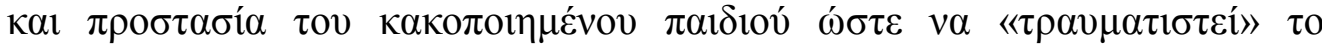

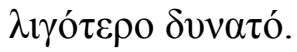

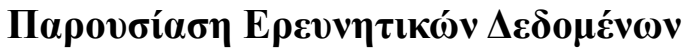

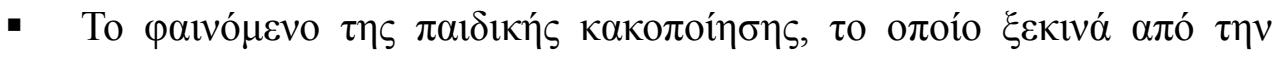

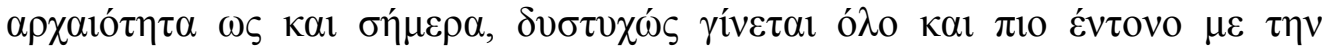

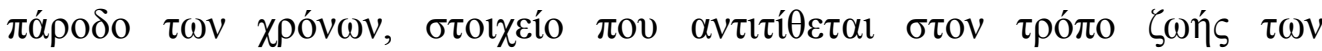

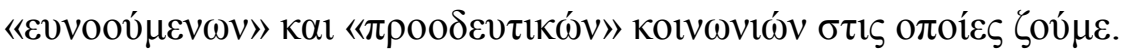




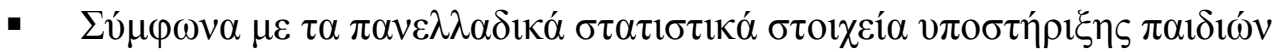

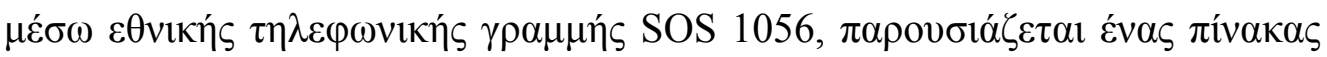

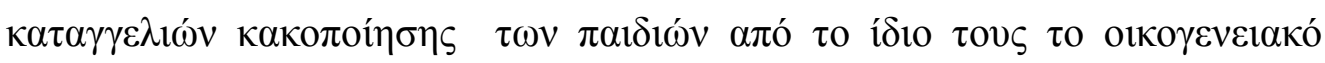

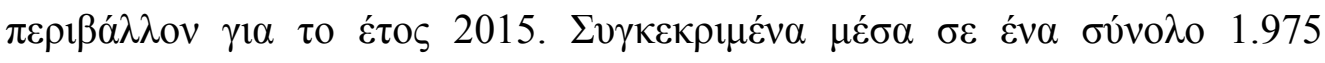
$\kappa \alpha \tau \alpha \gamma \gamma \varepsilon \lambda \iota \omega ́ v:$

\begin{tabular}{|c|c|c|c|c|}
\hline $\begin{array}{l}\text { H } \Lambda \text { IKIAKE } \\
\Sigma \text { OMA } \Delta \mathrm{E} \Sigma\end{array}$ & АГОРІА & 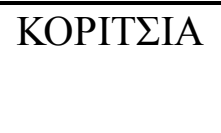 & $\begin{array}{l}\mathrm{A \Gamma \textrm {N } \Omega \Sigma \mathrm { TO }} \\
\Phi \mathrm{Y} \Lambda \mathrm{O}\end{array}$ & $\Sigma \mathrm{YNO} \Lambda \mathrm{O}$ \\
\hline 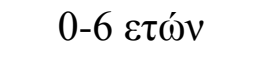 & 400 & 314 & 53 & $767(39 \%)$ \\
\hline 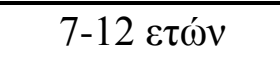 & 377 & 318 & 12 & $707(36 \%)$ \\
\hline 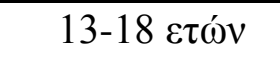 & 128 & 176 & 5 & $309(15 \%)$ \\
\hline $\begin{array}{l}\text { А } \gamma \nu \omega \sigma \tau \eta \\
\eta \lambda \iota \kappa i ́ \alpha\end{array}$ & 31 & 21 & 140 & $192(10 \%)$ \\
\hline $\begin{array}{l}\Sigma \mathrm{YNO} \Lambda \mathrm{O} \\
936\end{array}$ & $(47 \%) 829$ & $(42 \%) 210$ & $(11 \%) 1.975$ & $\begin{array}{l}1.975 \\
(100 \%)\end{array}$ \\
\hline
\end{tabular}

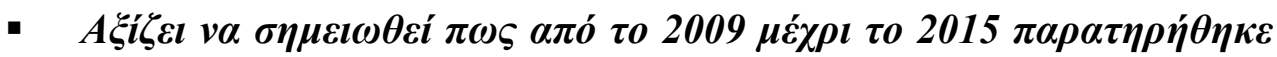

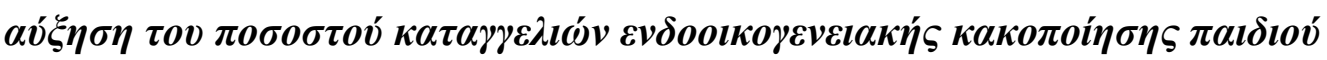

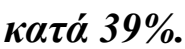

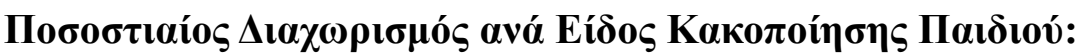

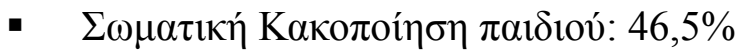

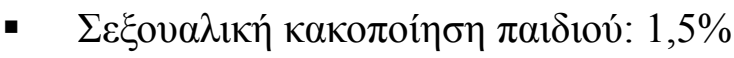

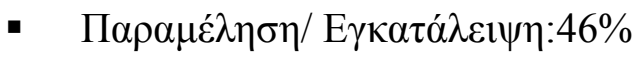

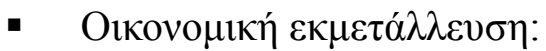

A) $\varepsilon \xi \dot{\omega} \theta \eta \sigma \eta \sigma \varepsilon \varepsilon \pi \alpha \imath \varepsilon \varepsilon i ́ \alpha: 1 \%$

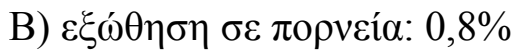

Г) $\varepsilon \mu \pi o ́ \rho 10 ~ \pi \alpha 1 \delta เ \omega ́ v: 0,5 \%$

$\Delta) \alpha \dot{\alpha} \lambda \mathrm{o}: 0,2 \%$

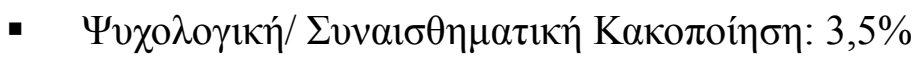




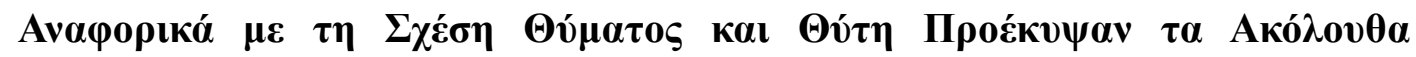

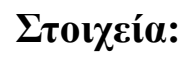

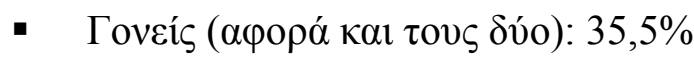

- M

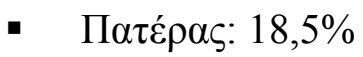

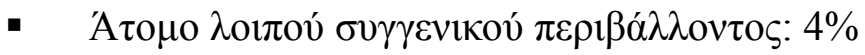

- $\quad$ A $\lambda \lambda 0: 4 \%$

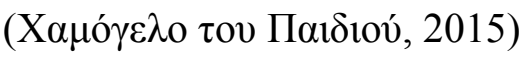

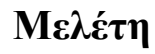

Пврі́ $\pi \tau \omega \sigma \eta \varsigma$

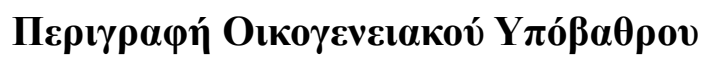

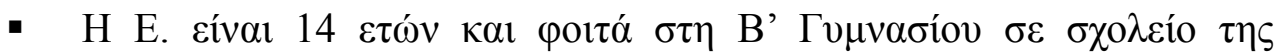

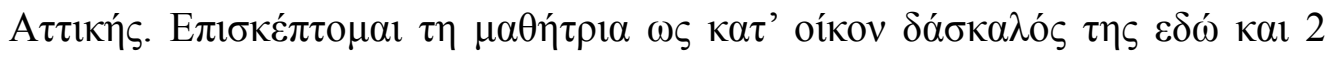

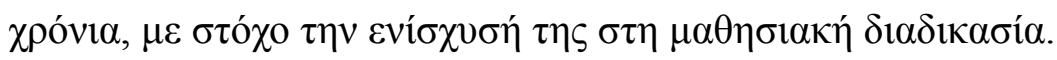

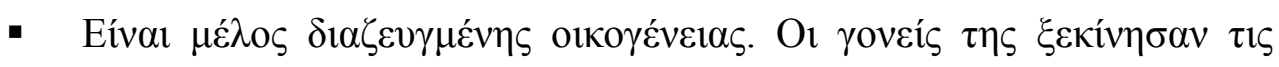

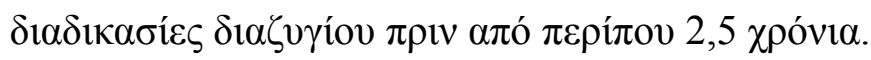

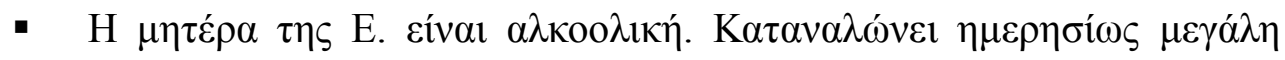

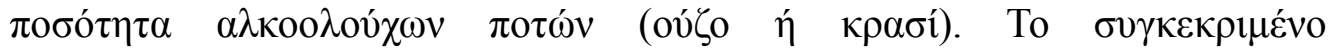

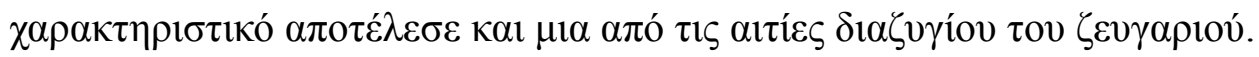

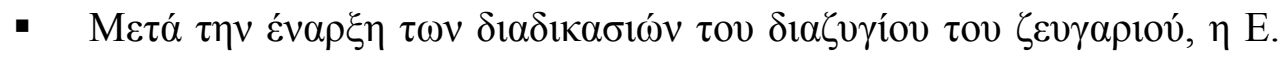

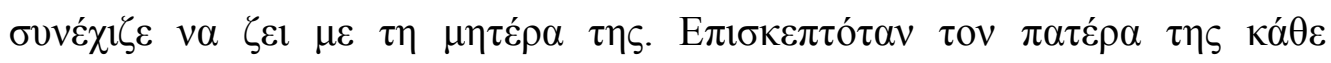

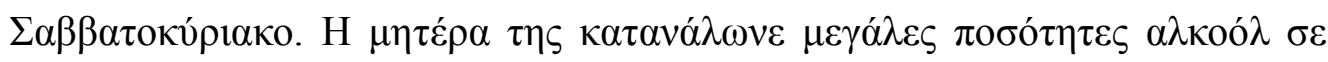

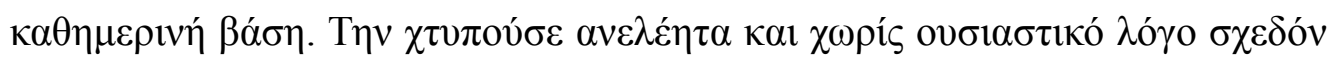

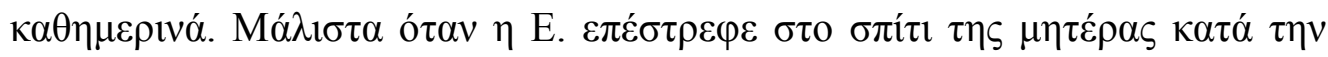

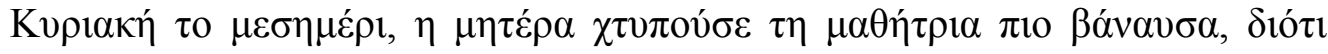

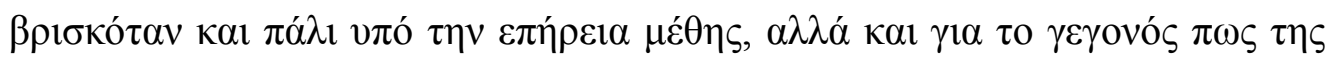

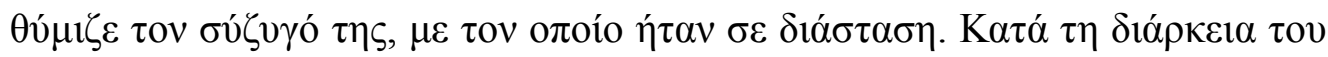

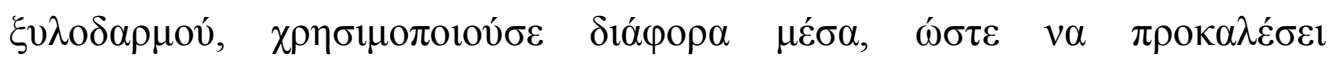

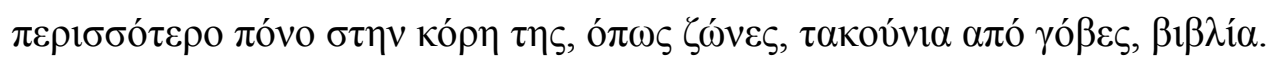




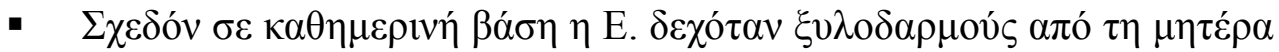

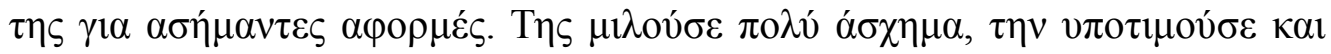

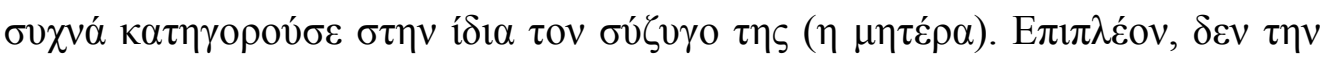

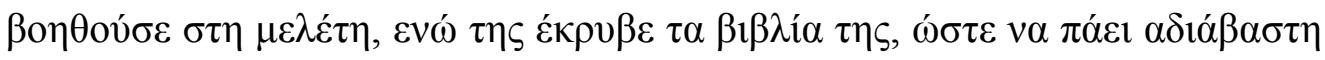

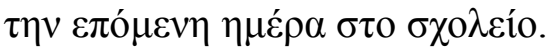

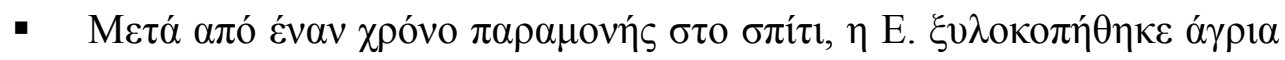

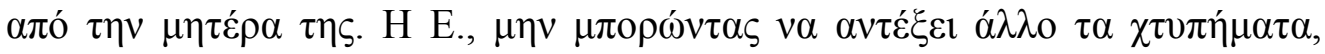

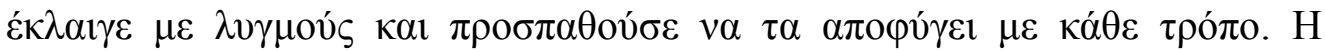

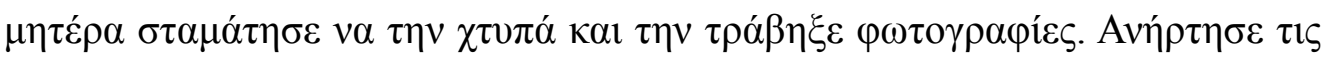

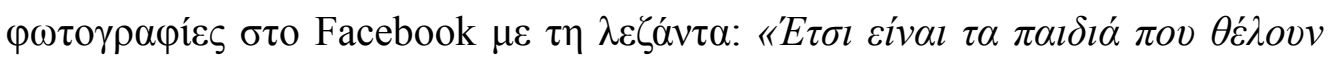

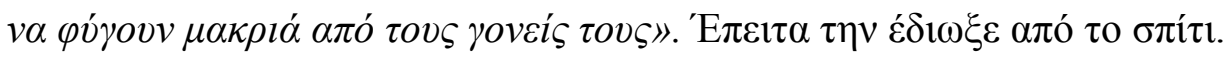

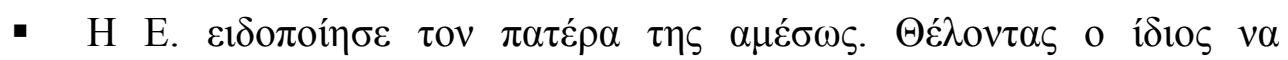

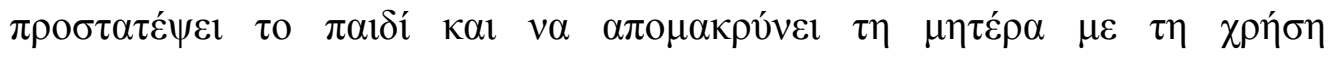

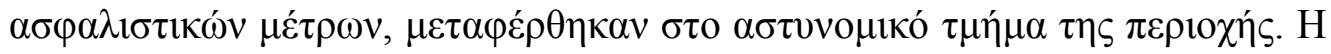

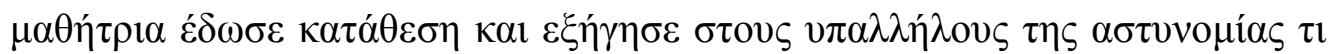

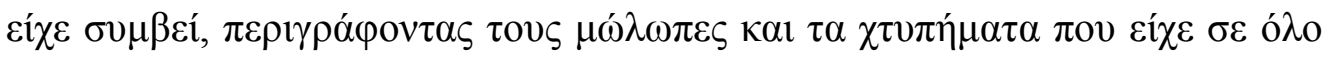
$\tau \eta \varsigma \tau о \sigma \omega ́ \mu \alpha$.

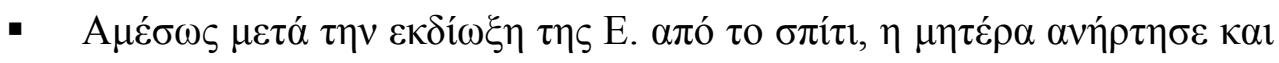

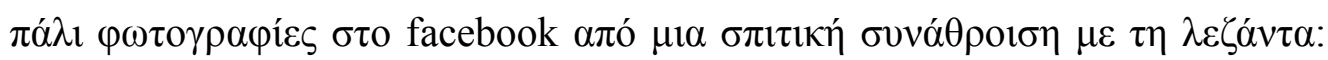

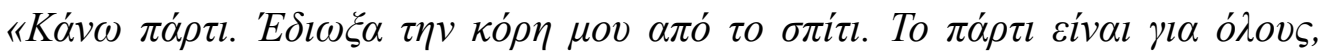
ó

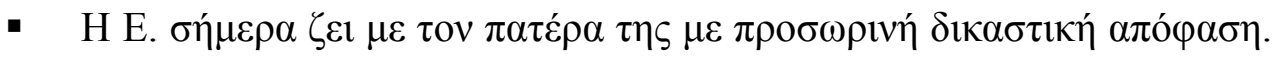

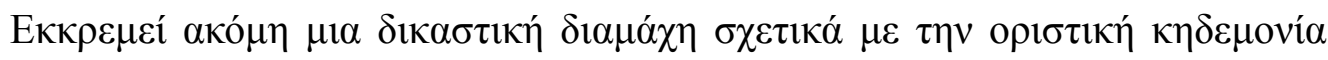

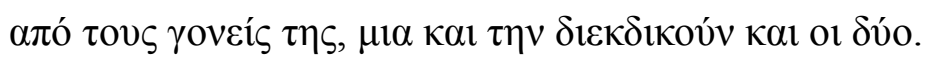

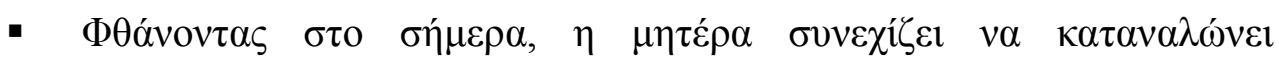

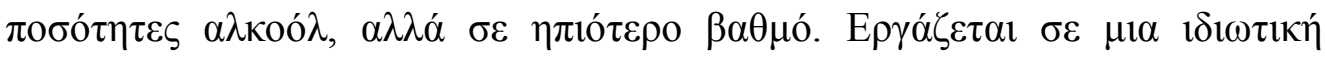

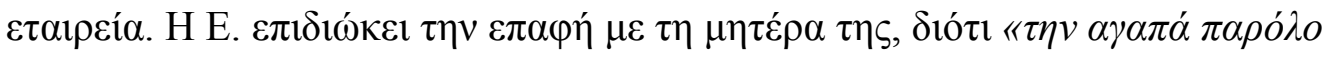

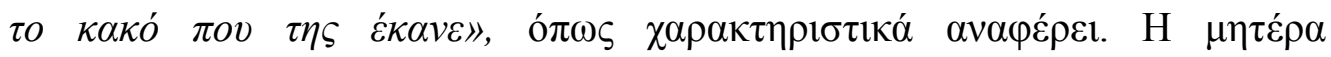

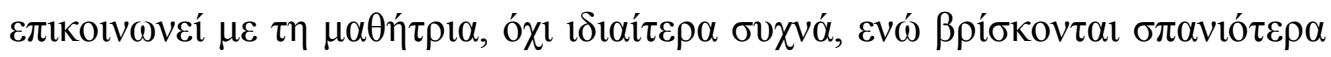

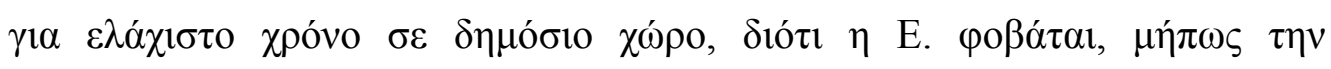

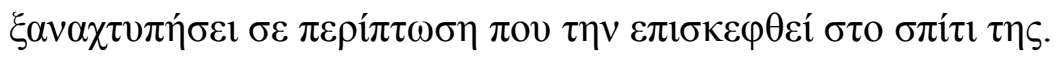




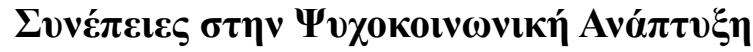

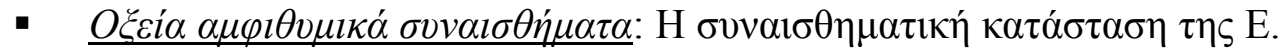

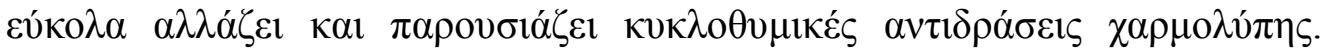

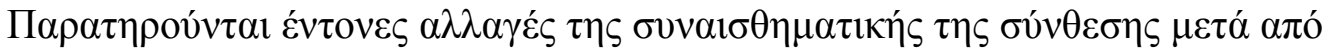

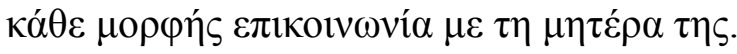

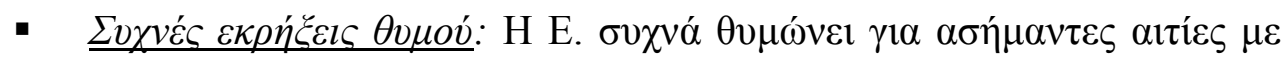

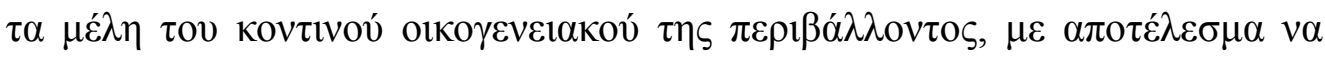

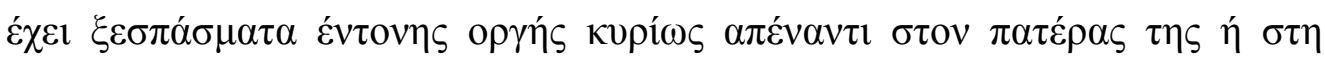

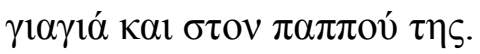

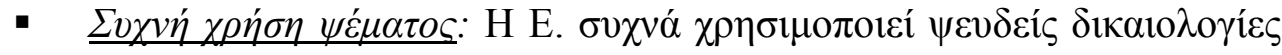

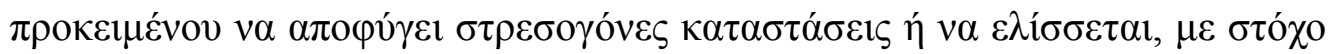

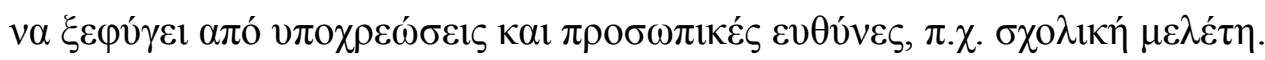

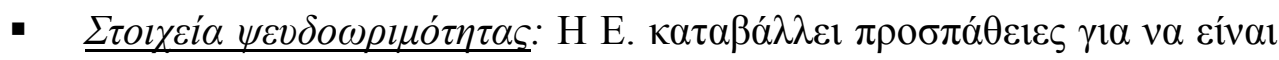

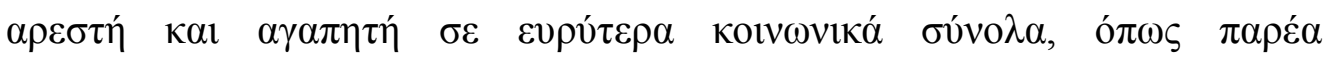

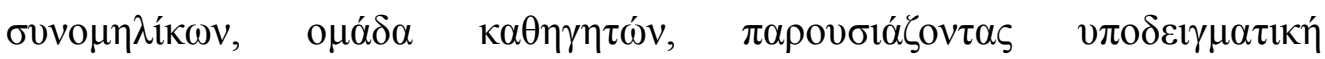

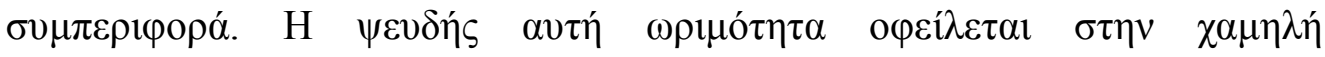

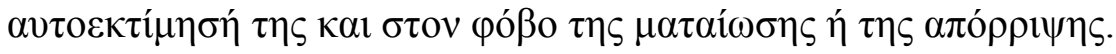

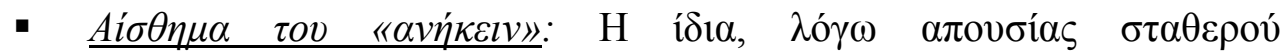

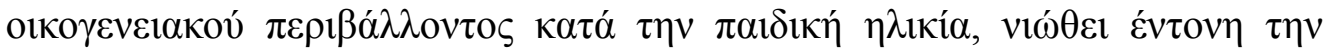

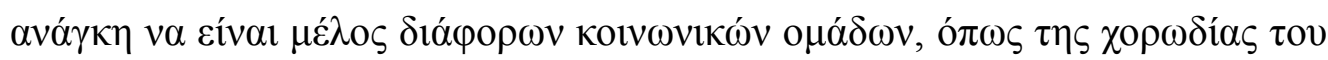

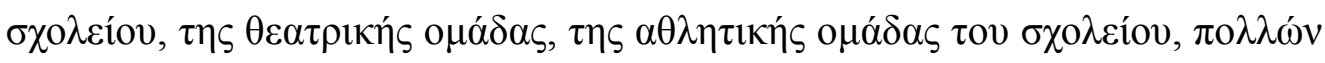
$\pi \alpha \rho \varepsilon \omega ́ v ~ \sigma v v o \mu \eta \lambda i ́ \kappa \omega v \kappa \lambda \pi$.

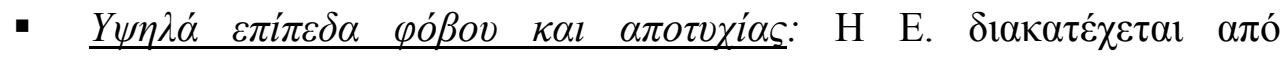

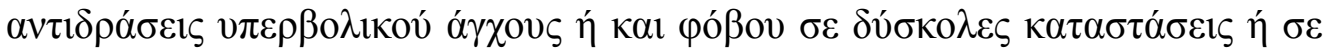

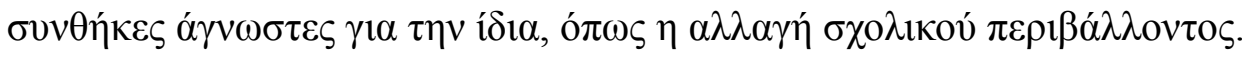

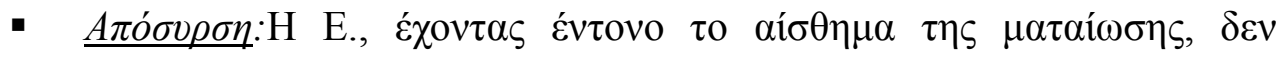

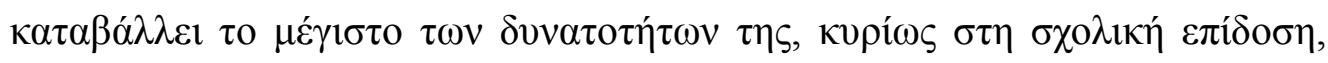

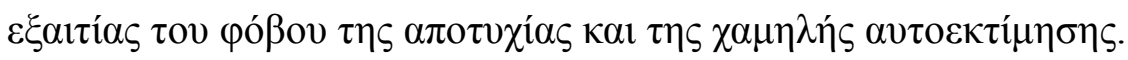

- Y Y

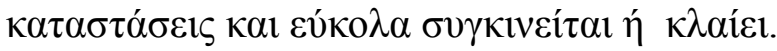

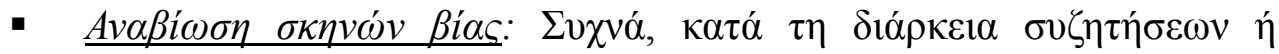

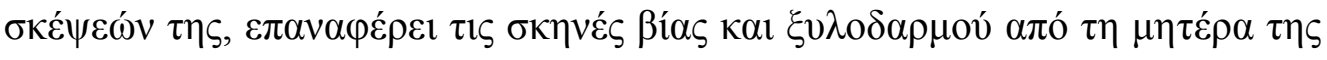

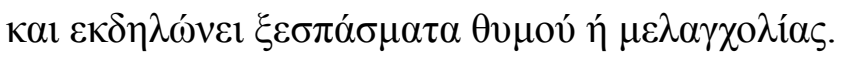




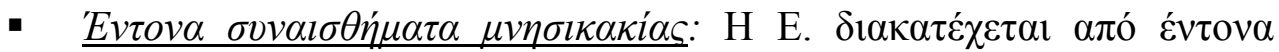

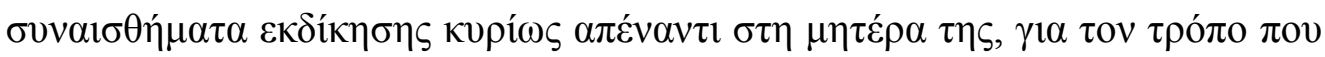

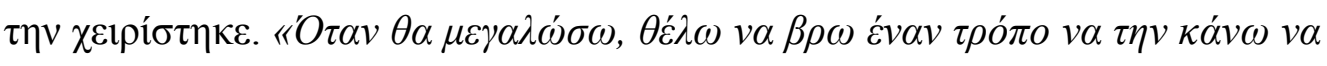

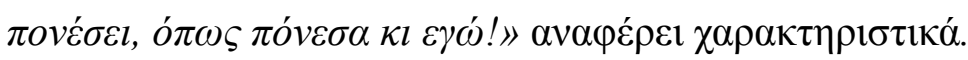

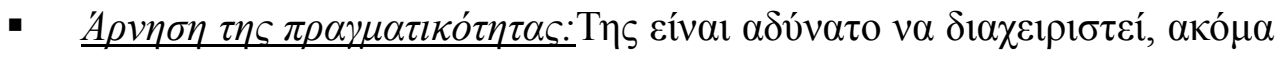

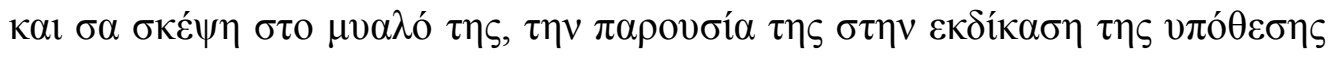

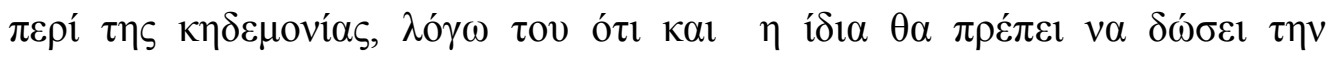

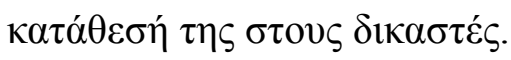

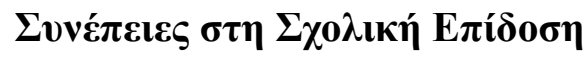

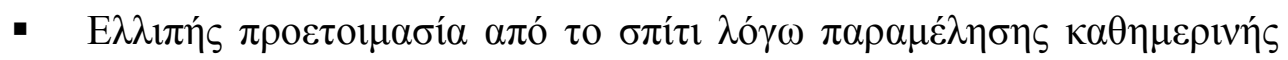
$\mu \varepsilon \lambda \varepsilon ́ \tau \eta \varsigma$.

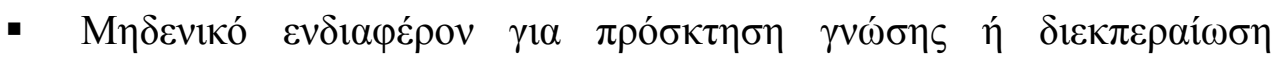

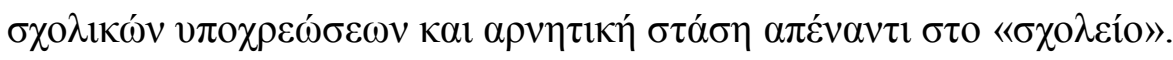

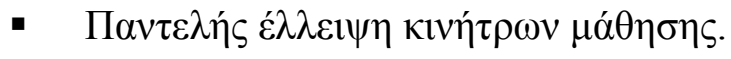

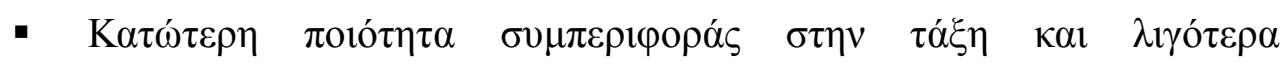

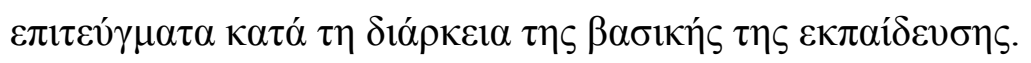

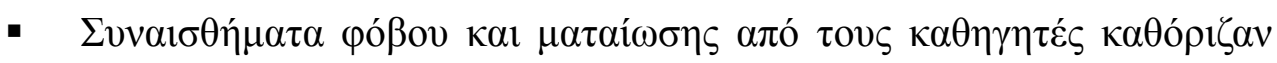

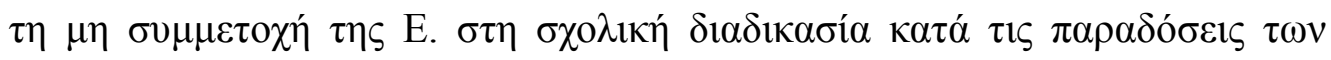
$\mu \alpha \theta \eta \mu \alpha ́ \tau \omega \nu \sigma \varepsilon \sigma \chi 0 \lambda 1 \kappa \varepsilon ́ \varsigma \omega ́ \rho \varepsilon \varsigma$.

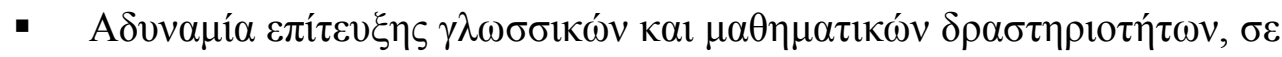

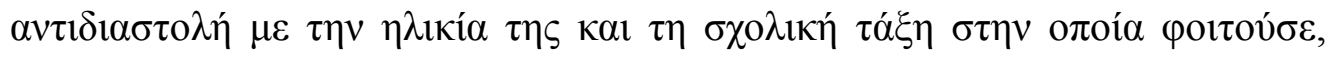

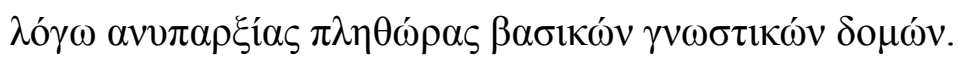

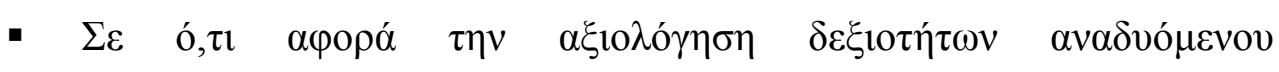

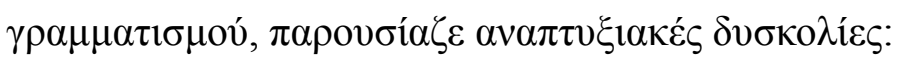

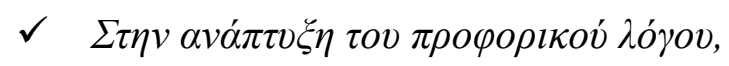

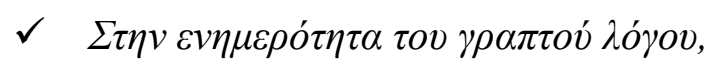

$\checkmark \quad \Sigma \tau \eta v \alpha v \alpha \gamma v \omega \sigma \tau \iota \kappa \eta \dot{~} \alpha \pi o \kappa \omega \delta \imath \kappa o \pi o i ́ \eta \sigma \eta, \varepsilon \pi \varepsilon \xi \varepsilon \rho \gamma \alpha \sigma i \alpha, \kappa \alpha \tau \alpha \nu o ́ \eta \sigma \eta$,

$\checkmark \quad \Sigma \tau \eta v \pi \alpha \rho \alpha \gamma \omega \gamma \eta \dot{~ \gamma \rho \alpha \pi \tau o v ́ ~ \lambda o ́ \gamma o v . ~}$

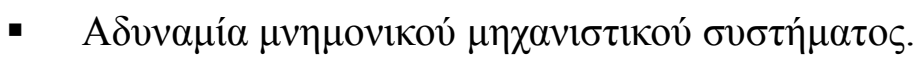

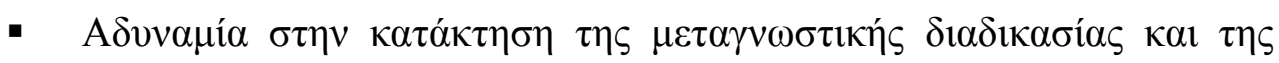

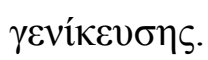




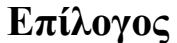

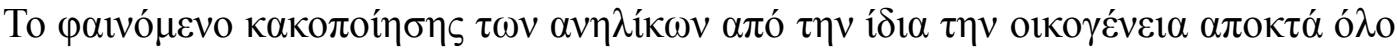

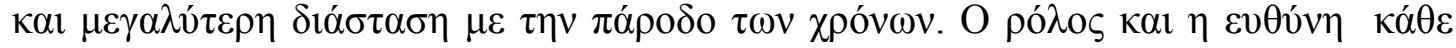

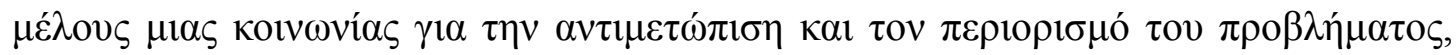

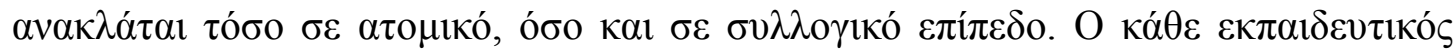

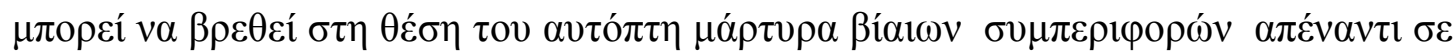

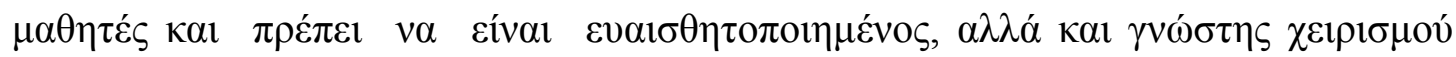

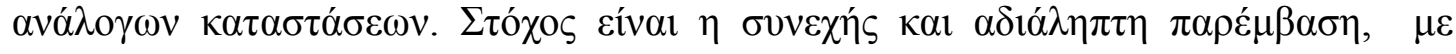

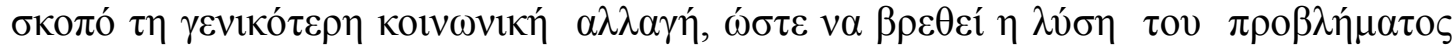

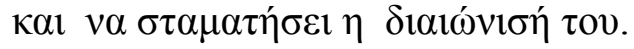

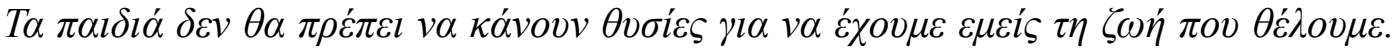

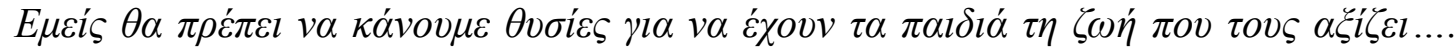

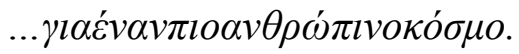

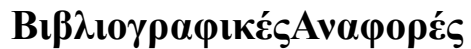

Jaffle, P., Wolf, D., Wilson, S. \& Zak, L. (1986) Similarities in Behavioural and Social Maladjustment Among Child Victims and Witnesses to Family Violence. American Journal of Orthopsychiatry

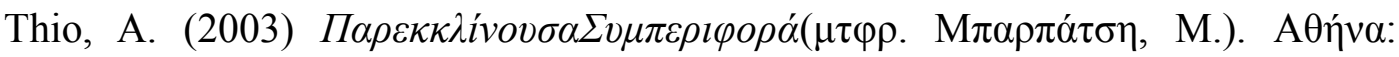
'E $\lambda \lambda \eta v$.

World Health Organization, (1999) Report of the Consultation on Child Abuse Prevention. Geneva: W.H.O.

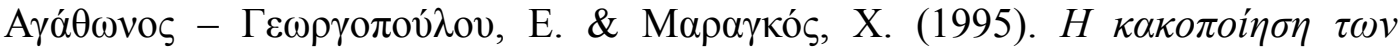

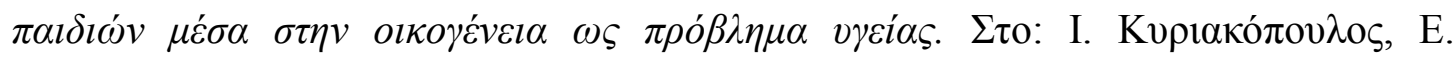

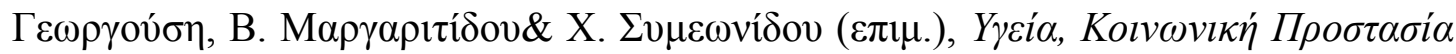

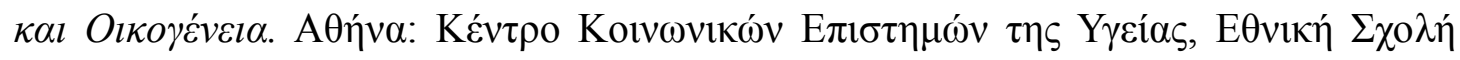
$\Delta \eta \mu o ́ \sigma ı \alpha \varsigma$ Y Y̌ías.

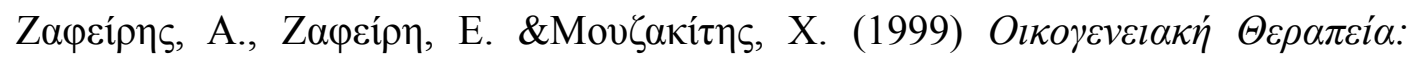

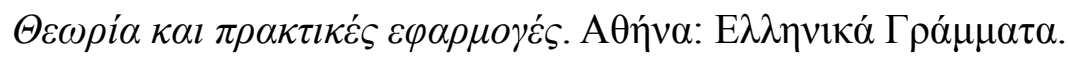




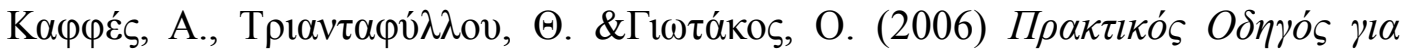

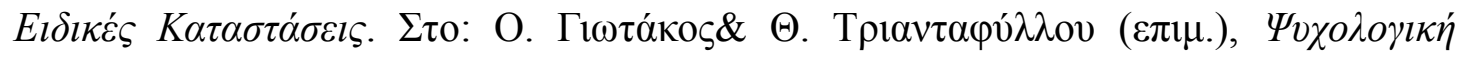

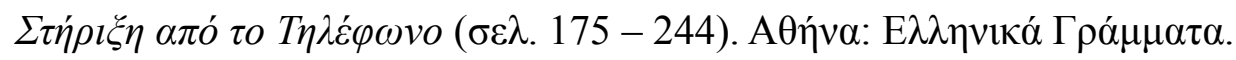

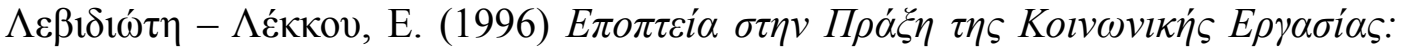

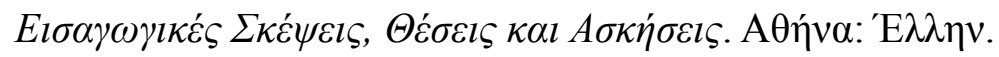

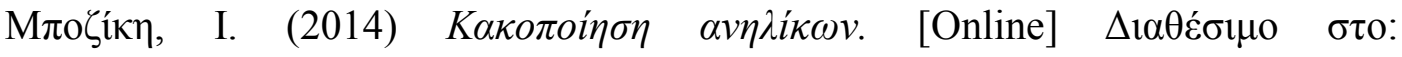

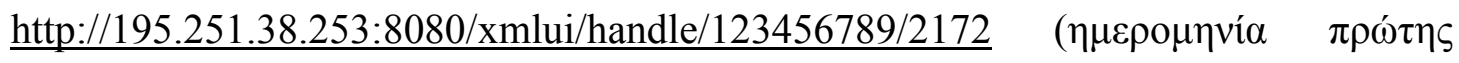

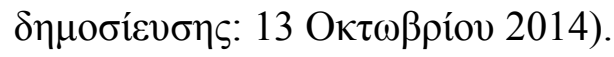

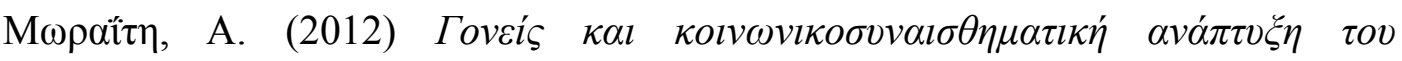

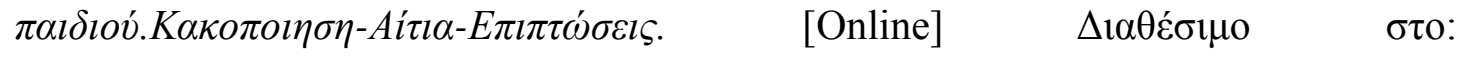
http://apothetirio.teiep.gr/xmlui/bitstream/handle/123456789/774/psa 2012019.pdf?se quence $=1$ (

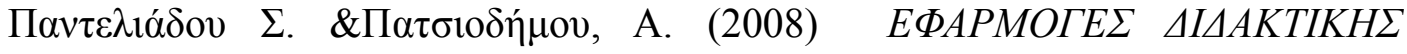

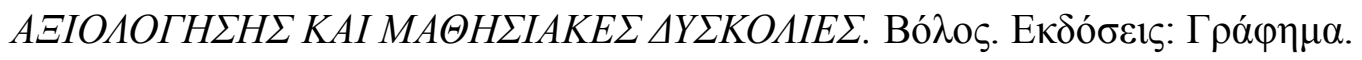

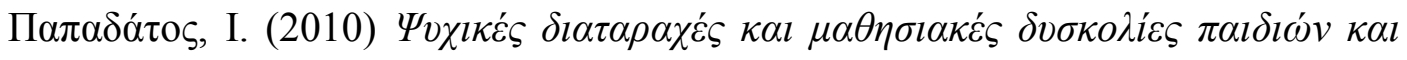

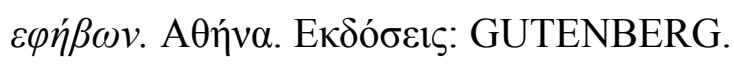

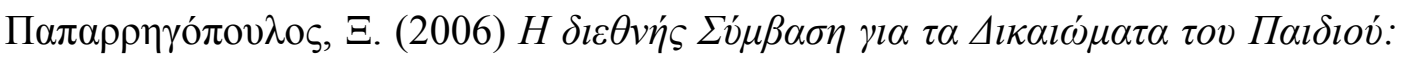

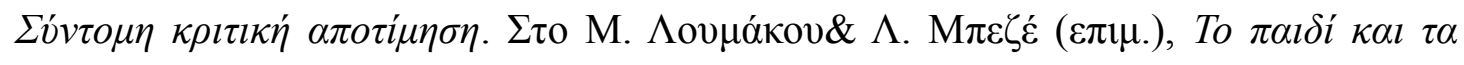

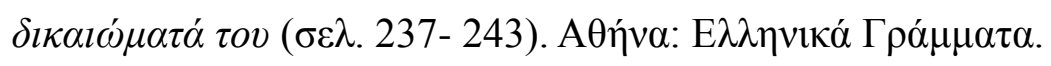

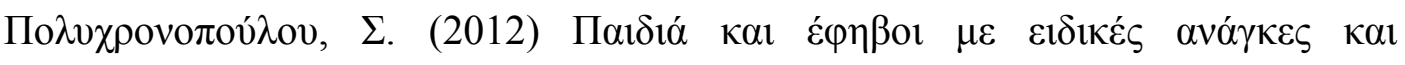

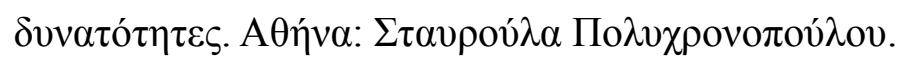

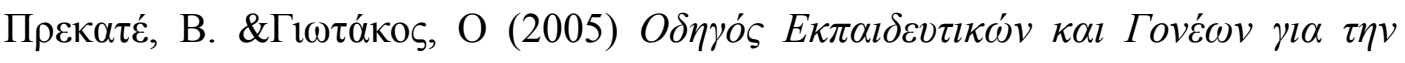

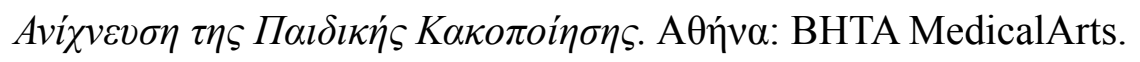

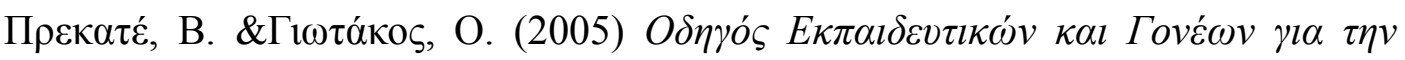

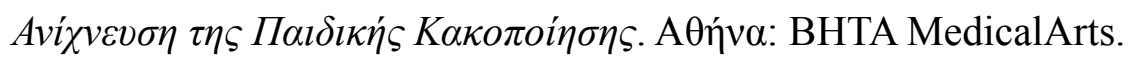

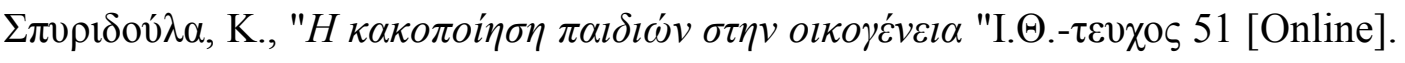

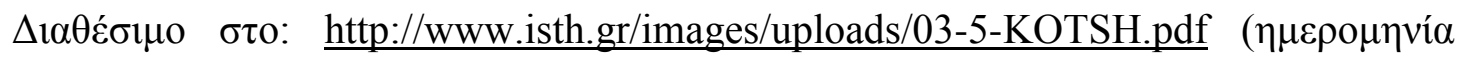

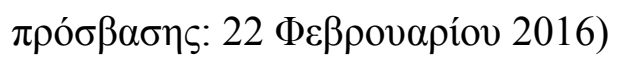




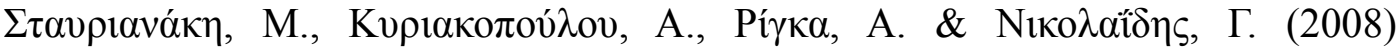

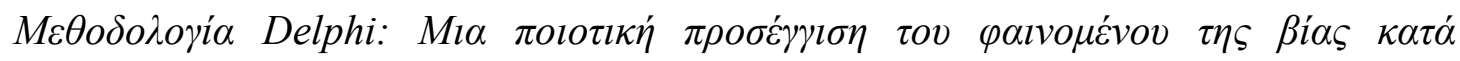

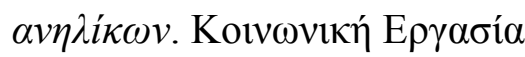

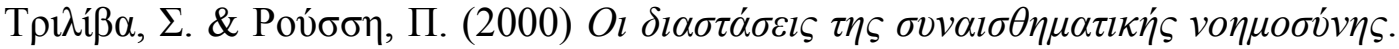

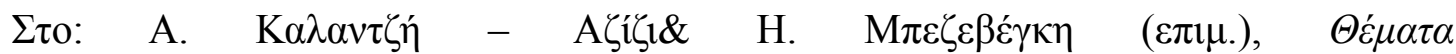

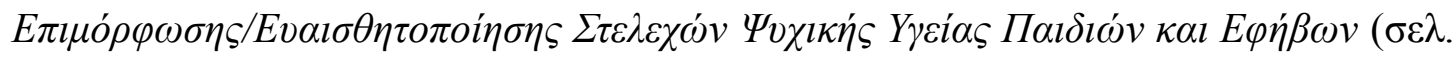

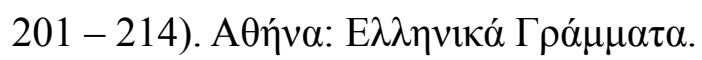

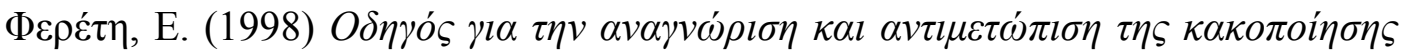

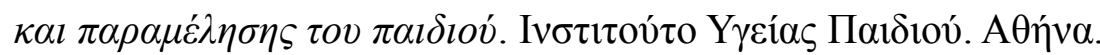

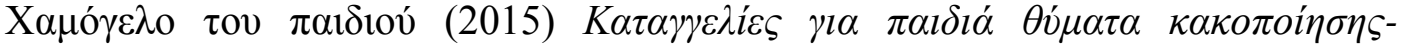
$\pi \alpha \rho \alpha \mu \varepsilon ́ \lambda \eta \sigma \eta \varsigma$. [Online] $\Delta \mathrm{t} \alpha \theta \dot{\sigma} \sigma \mu \mathrm{o} \sigma \tau \mathrm{o:} \mathrm{http://www.hamogelo.gr/53.1/Kataggelies-gia-}$

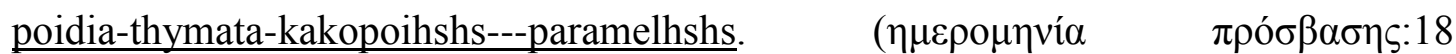
Фєßрovapíov 2016). 